\title{
Cognitive load theory as an aid for instructional design
}

\section{Graham Cooper}

This paper attempts to draw together several recent findings in educational psychology that have led to the development and application of cognitive load theory to the format of instruction (Chandler and Sweller, unpublished manuscript; Sweller, 1988). These findings are directly related to the processes involved in learning, and will ultimately affect the way instructional design is approached.

Cognitive load may be viewed as the level of 'mental energy' required to process a given amount of information. As the amount of information to be processed increases, so too does the associated cognitive load. Cognitive load theory suggests that effective instructional material promotes learning by directing cognitive resources towards activities that are relevant to learning rather than to processes that are an adjunct to learning.

Cognitive load theory grew out of research investigating the differences between experts and novices. This research will be discussed briefly before returning to the issue of cognitive load theory.

\section{Differences between an expert and a novice}

The primary role of an instructor is to transform a novice into an expert within a given subject area. Much of the research within cognitive science has investigated the differences between expert and novice problem solvers. It is not surprising that 'experts' solve problems faster, and with fewer errors, than 'novices' (Sweller and Cooper, 1985). These represent the behavioural (or performance) differences that any professional instructor is likely to have encountered. But instructors need to look beyond such observable differences in problem solving performance. One needs to identify the cognitive phenomenon which give rise to the speed and accuracy of expert performance. 
The essential determinant of expert performance appears to be schemes (Gick and Holyoak, 1980; 1983) "A schema is defined as a mental construct permitting problem solvers to categorise problems according to solution modes" (Sweller, Chandler, Tierney, and Cooper, 1990, p176). Schemas enable experts to categorise problems and consequently solve them. Novices, not possessing schemes, are unable to categorise problems. Novices have no alternative other than to engage in general search techniques such as trial-and-error, or means-ends analysis (Chi, Glaser, and Rees, 1982; Larkin, McDermott, Simon and Simon, 1980).

\section{Cognitive load theory}

If schemes are the primary determinants of expertise, then good instructional design may be defined as that which facilitates schema acquisition. Schemas may said to have been acquired once they have been successfully stored into long term memory for later retrieval and application. Note, however, that schemes may only be acquired by being attended to, and processed by, the cognitive resources available in working memory.

Cognitive load theory (Sweller, 1988) suggests that effective instructional material facilitates learning by directing cognitive resources towards activities that are relevant to schema acquisition. Instructional material that requires trainees to direct cognitive resources to activities other than schema acquisition may thus be viewed as inferior. Two broad areas of application will be discussed. These are the use of worked examples, and the integration of multiple sources of information into a unified presentation.

\section{The use of worked examples}

Cognitive load theory suggests that learning within a problem solving domain is facilitated by a heavy use of worked examples rather than a heavy use of conventional problem solving (Sweller and Cooper, 1985; Cooper and Sweller, 1987). The presentation of worked examples enables students to dedicate their cognitive resources to recognising and remembering problem structures and applications of rules as demonstrated by the examples. Conversely, conventional problem solving requires students to engage in search strategies of the problem space to 'discover' for oneself the problem structure and application of the rules.

'Trial and error' is one example of a search strategy, but the most common search strategy is 'means-ends analysis'. Means-ends analysis operates by working backwards from the problem goal to the initial information given (Larkin, McDermott, Simon, and Simon, 1980). Means-ends analysis is very effective in obtaining solutions to problems but it imposes a relatively high cognitive load and consequently reduces the cognitive resources 
available for learning (Owen and Sweller, 1985; Sweller and Levine, 1982; Sweller, 1988).

\section{Integration of multiple sources of information}

When worked examples require both text and diagrammatical information to be presented then the text (as far as possible) should be integrated into the diagram rather than sitting above, below, or beside the diagram as a dual (or split) source of information.

Students presented a split source of information will need to expend a portion of their cognitive resources mentally integrating the different sources of information. This reduces the cognitive resources available for learning.

Figure 1: Split source format of instruction

\section{INSULATION RESISTANCE TESTS}

\section{a) CONDUCTORS IN PERMANENT WIRING}

Test: $\quad$ To test Insulating Resistance from conductors to earth

How i) Disconnect appliances and busways during these tests. Make sure Conducted: main switch is "on" and all fuses are "in". Remove main earth from neutral bar and set meter to read insulation. Connect one lead to earth wire at MEN bar and take first measure by connecting the other lead to the active. Take next measure by connecting the lead to the neutral.

ii) If resistance is not high enough in either of the two tests in i) then measure each circuit separately.

Results i) At least One Megaohm

required: ii) Same result as i) above

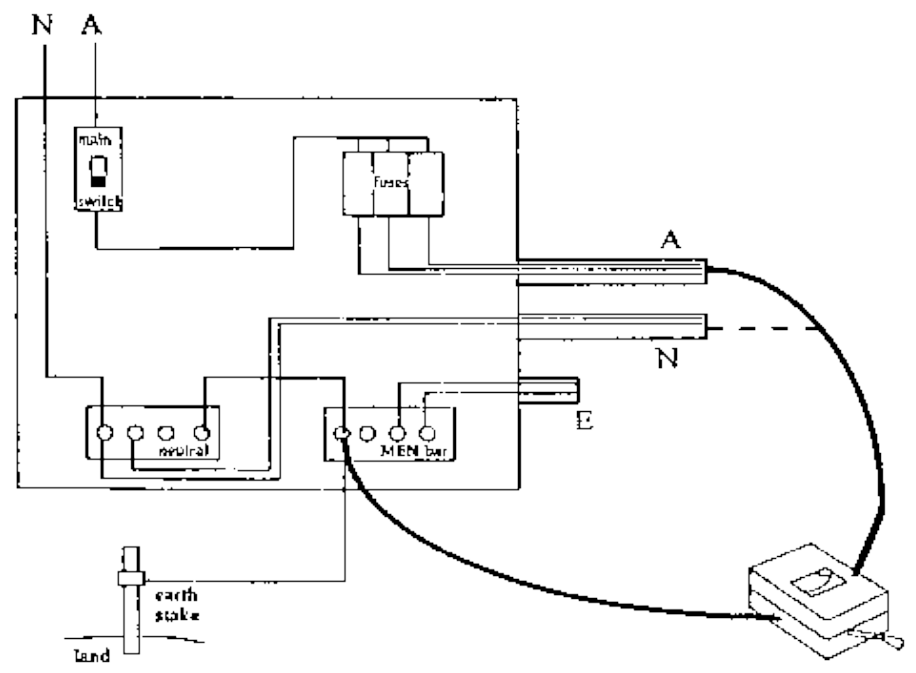


Conversely, an integrated format clearly demonstrates the relation between text and diagrams, thus reducing the cognitive load required to process the total information presented within the example. Cognitive resources are then free to attend to learning the new information (Tarmizi and Sweller, 1988; Sweller, Chandler, Tierney, and Cooper, 1990).

The superiority of integrated formats over split source presentations has recently been demonstrated for initial instructional material (Chandler and Sweller, unpublished manuscript). Chandler and Sweller tested the usefulness of integrated formats in the area of elementary electrical engineering. A split source of text and diagrammatical information (figure 1) was compared against an integrated format presenting equivalent information (figure 2).

Figure 2: Integrated format of instruction

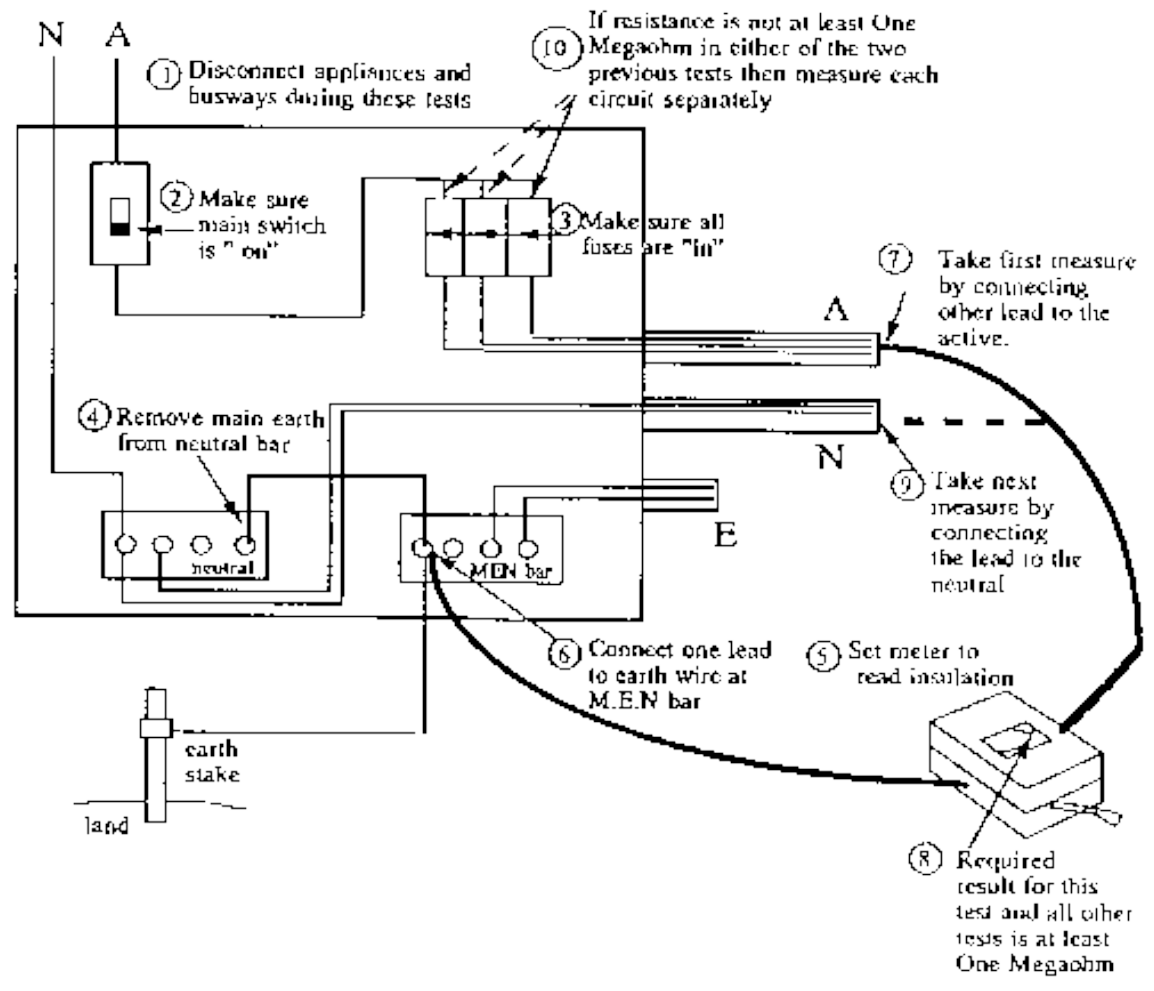


Trainees presented the integrated format displayed significantly greater learning of the presented material than did trainees presented the information in a split source format. The enhancement in learning was displayed in both written tests of theory, and practical tests of application. Moreover, the superior performance of the integrated format group was observable both immediately after training had completed, and also three months later in follow-up tests.

It should also be noted that a single source of integrated text facilitates greater learning than multiple sources of text conveying equivalent information (Sweller, Chandler, Tierney, and Cooper, 1990).

Finally, instructional designers need to ensure the eradication of non essential ("nice-to-know") information from instructional material. This is consistent with cognitive load theory. Attending to (and processing of) the non essential information reduces the cognitive resources that may be given to attending to the essential information. Also, Chandler and Sweller (unpublished manuscript) suggest that if text and diagrammatical information say the same thing (ie. one is redundant) then only one form of instruction should be presented - either text or diagram, but not both, as this too imposes an additional cognitive load. Learning of essential material is enhanced by eradicating all non essential information.

\section{References}

Chandler, P. and Sweller, J. Cognitive Load Theory and the Format of Instruction. (unpublished manuscript; under review for publication in Cognition and Instruction).

Chi, M., Glaser, R. and Rees, E. (1982). Expertise in problem solving. In R. Sternberg (Ed.), Advances in the psychology of human intelligence. Hillsdale, N.J.: Erlbaum, (pp. 7-75).

Cooper, G. and Sweller, J. (1987). The effects of schema acquisition and rule automation on mathematical problem-solving transfer. Journal of Educational Psychology, 79, 347-362.

Gick, M. L. and Holyoak, K. J. (1980). Analogical problem solving. Cognitive Psychology, 12, 306-355.

Gick, M. L. and Holyoak, K. J. (1983). Schema induction and analogical transfer. Cognitive Psychology, 15,1-38.

Larkin, J., McDermott, J., Simon, D. and Simon, H. (1980). Models of competence in solving physics problems. Cognitive Science, 4, 317-348.

Owen, E. and Sweller, J. (1985). What do students learn while solving mathematics problems. Journal of Educational Psychology, 77, 272-284.

Sweller, J. (1988). Cognitive load during problem solving: Effects on learning. Cognitive Science, 12, 257-285.

Sweller, J., Chandler, P., Tierney, P. and Cooper, M. (1990). Cognitive load and selective attention as factors in the structuring of technical 
material. Journal of Experimental Psychology: General, 119, 176-192.

Sweller, J. and Cooper, G. A. (1985). The use of worked examples as a substitute for problem solving in learning algebra. Cognition and Instruction, 2, 59-89.

Sweller, J. and Levine, M. (1982). Effects of goal specificity on means-ends analysis and learning. Journal of Experimental Psychology: Learning, Memory, and Cognition, 8, 463-474.

Tarmizi, R. and Sweller, J. (1988). Guidance during mathematical problem solving. Journal of Educational Psychology, 80, 424-436.

Please cite as: Cooper, G. (1990). Cognitive load theory as an aid for instructional design. Australian Journal of Educational Technology, 6(2), 108113. http: / / www.ascilite.org.au/ajet/ajet6/ cooper.html 